StricklaNd, W. N. (1960). J. gen. Microbiol. 22, 583-588

\title{
A Rapid Method for Obtaining Unordered Neurospora Tetrads
}

\author{
BY W. N. STRICKLAND \\ Department of Biological Sciences, Stanford Unizersity, Stanford, California, U.S.A.
}

SUMMARY : A method of collecting unordered asci of Neurospora crassa makes use of the natural ejection of the ascospores from perithecia in groups of eight. Genetic evidence from 2374 groups shows that they were derived from individual asci. The method is reliable and efficient.

Genetic tetrad data in Neurospora have almost exclusively been obtained from ordered asci (e.g. Lindegren \& Lindegren, 1942; Houlahan, Beadle \& Calhoun, 1949; Barratt, Newmeyer, Perkins \& Garnjobst, 1954). However, for many genetic purposes ordered asci are unnecessary. This is true of investigations concerning chromatid interference. In the course of experiments with tetrads to evaluate interference in Neurospora crassa, it was recently observed that under suitable conditions the ascospores from individual asci are forcibly ejected in a group and that this group can be recovered from an apposed surface. This method has proved reliable and efficient and has already been used for collecting interference data from 2616 tetrads in multiply-marked crosses. After most of these data had been collected, it was pointed out by Dr N. H. Horowitz that the first tetrads ever to be analysed genetically in Neurospora were obtained by an exactly similar method (Shear \& Dodge, 1927, p. 1032). The present paper describes the method and presents genetic evidence of its reliability.

\section{METHODS}

Preparation of crosses. The protoperithecial parent was inoculated on a Petri plate containing 30-35 ml. supplemented defined crossing medium in $2 \%(w / v)$ agar (Westergaard \& Mitchell, 1947). Generally the protoperithecial parent was selected for lack of aerial hyphae which might obstruct the free flight of ejected ascospores. However, wild-type has been successfully used as the protoperithecial parent by Dr D.D. Perkins. After incubation for 6 days in the dark at $25^{\circ}$, the protoperithecial parent was fertilized and re-incubated in the dark at $25^{\circ}$. The crosses used so far have begun to shoot ascospores about 8-9 days after fertilization and have continued to shoot effectively for 10-14 days.

Collection of unordered asci. The ejected spores were caught on a $4 \%(w / v)$ agar surface placed a few millimetres away from the ostioles of the perithecia in the lid of an inverted Petri dish. Many of the spores were recovered as more or less tightly clustered groups of eight. Before the agar surface becomes overcrowded with ascospores, it must be replaced by another. This may occur in a period ranging from a few seconds onwards, depending upon the particular cross, and upon physiological and environmental conditions 
Factors which affect spore ejection and the grouping of spores. Controlled experiments have not been carried out to determine the relative importance of such factors as light, humidity and age of the cross. Observations suggest, however, that such factors do affect both the rate of ascospore ejection and the efficiency of grouping.

When crosses were first brought out of the dark the rate of spore ejection was very low, but it consistently increased very rapidly after the cross was placed in the light. On returning the cross to the dark, the ejection rate slowed down and after $12 \mathrm{hr}$. was again very low.

The age of the cross also affected the rate of spore ejection. When the perithecia began ejecting spores on the eighth or ninth day after fertilization, the rate was low. The rate then increased and reached a maximum after three or four days, after which it slowly declined.

The efficiency of the grouping was influenced by the distance between the ostioles and the receiving agar surface; the grouping was more efficient when this distance was kept to a minimum.

The compactness of the groups must also be influenced by the angle between the ostiole and the receiving agar surface. The groups should be most compact when that angle is $90^{\circ}$ and should become less and less compact as the angle decreases. When shooting first started, the ostioles were clear and the ascospores were not obstructed in their flight. However, as the crosses aged, stray ascospores and other debris tended to clog up the ostioles with a consequent decrease in the efficiency of grouping.

Humidity also seemed to affect the grouping. This conclusion is based on the fact that when wet agar surfaces were used to catch the ejected spores, the grouping gradually became looser and eventually the spores seemed to be shot quite at random.

Handling and storage of ascospores. It was found that the ascospores ejected in the groups were immature at the time of ejection and when heat-shocked immediately, they did not germinate. The degree of germination increased enormously when the ascospores were stored on the surface of supplemented medium for 6 days.

The $4 \%(\mathrm{w} / \mathrm{v})$ agar surface in the vicinity of a shot group of eight ascospores was soaked with a sodium hypochlorite solution (200 p.p.m. available chlorine) and the individual ascospores were spread over this area. They were then isolated individually on to supplemented medium in $75 \mathrm{~mm}$. diam. test tubes and stored for 6 days at $25^{\circ}$. An experiment designed to find the best period of storage after shooting showed $0 / 80$ ascospores germinated when heat-shocked immediately after ejection, $73 / 80$ ascospores germinated when heat-shocked after storage for 3 days, and 76/80 when heat-shocked after storage for 6 days.

The degree of hydration also affects germination. Random spores from one of the crosses were collected over a period of 7 days and stored under two sets of conditions: on a completely dry glass surface, and on a wet agar surface. It is obvious from the results (Table 1) that storage under dry conditions followed by heat-shock without re-hydration decreased the degree of germination drastically. Therefore, the groups of eight spores were stored on freshly made supplemented medium for 6 days before heat-shocking. 
Table 1. The effect of dehydration on the germination of ascospores of

Neurospora from the cross inos pab-1 bis a $\times$ hist- $1 \mathrm{~A}$

\begin{tabular}{|c|c|c|}
\hline \multirow{2}{*}{$\begin{array}{c}\text { Period of } \\
\text { storage } \\
\text { (days) }\end{array}$} & \multicolumn{2}{|c|}{ \% germination* } \\
\hline & Stored dry & Stored wet \\
\hline 0 & $72 \cdot 8$ & $75 \cdot 9$ \\
\hline 6 & $91 \cdot 0$ & $92 \cdot 3$ \\
\hline 12 & $68 \cdot 0$ & $95 \cdot 2$ \\
\hline 18 & $44 \cdot 2$ & $93: 0$ \\
\hline 24 & $19 \cdot 4$ & 91.9 \\
\hline 27 & $30 \cdot 8 \dagger$ & $94 \cdot 4$ \\
\hline
\end{tabular}

* Measured on samples of not less than 500 ascospores.

$\dagger$ A sample of $\mathbf{5 8 0}$ ascospores which had been stored dry for $\mathbf{2 7}$ days was soaked in water for $24 \mathrm{hr}$. before heat-shocking. The degree of germination then rose to $97.5 \%$.

\section{RESULTS}

The method has been used with the crosses shown in Table 2. So far 2616 asci have been analysed and of these 2374 were fully classifiable. In this total there were only 24 groups (about $1 \%$ ) which gave irregular ratios for one or more pairs of alleles. Twenty-two of these groups occurred in the class in which four genotypes were recovered and the other two occurred in the class in which three genotypes were recovered. Eleven of these irregularities may have resulted from picking up fortuitous groups of eight spores originating in more than one ascus, while the other thirteen more probably resulted from such events as gene conversion and extra-mitotic division (see Emerson, 1956).

The twenty-four irregular groups shown in Table 2 can be split up into subclasses ('Table 3). Four of the irregular groups in cross 5 had 3:1 or 1:3 ratios for all seven pairs of alleles and so might have been the result of extra-mitotic divisions. There were six groups which were irregular with respect to aurescent but which gave normal 1:1 ratios for all the other markers in the cross. Four of these occurred in close sequence, suggesting a clonal origin. These six groups may have resulted from mutation or gene conversion. Crosses 4-6 were used in an analysis of interference (unpublished observations), and only biscuit and aurescent were completely scored in all asci. The other markers were only partially scored unless a double recombination product or an irregular segregation had occurred in an ascus; yellow was not recorded in all asci because it is laborious to score in a biscuit-aurescent background.

Three of the groups with irregular allele ratios in cross 5 involve inositol and histidine, in spite of the fact that these markers were scored completely only in a fraction of the population. These three cases were detected because they were associated with reciprocal exchanges, leading to the appearance of double recombination products. These apparent double recombination products were then scored for the other markers. It was found that the group with the 3 hist $^{+}: 1$ hist $^{-}$was associated with a reciprocal exchange in the bis-pab-2 interval while the two groups with 3 inos $^{-}: 1$ inos $^{+}$contained exchanges in both the inos-bis and the bis-pab-2 intervals. The remaining eleven groups were a conglomeration of different genotypes and the simplest explanation is that they arose from picking up a fortuitous group of eight ascospores. They constituted less than $\frac{1}{2} \%$ of the total. 


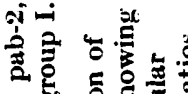

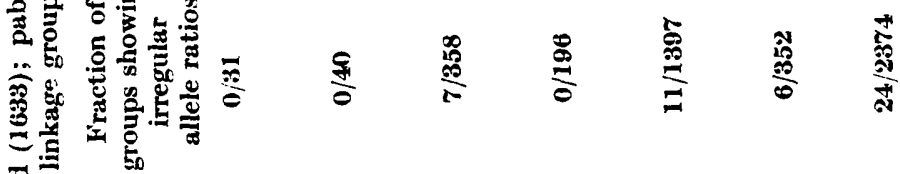

है 


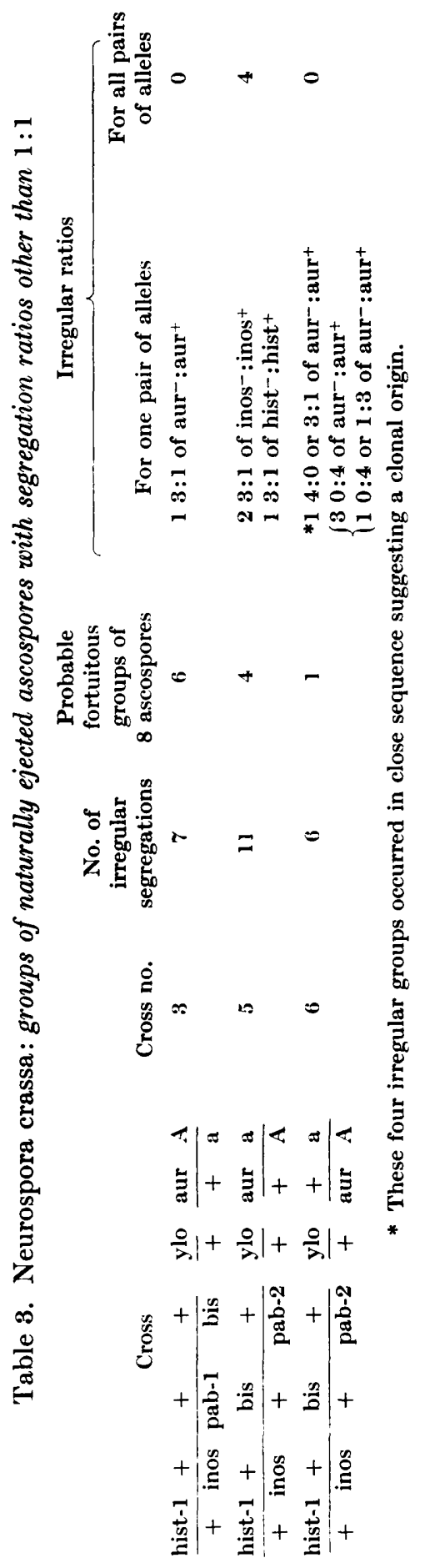




\section{DISCUSSION}

The results show that the method of recovering asci as groups of normally ejected ascospores is very reliable and efficient. Even if all 24 irregular asci are attributed to errors of method, the technique can be used perfectly well for projects such as an analysis of interference. For an analysis of rare events such as gene conversion or recombination in a very short region, the technique could probably be used if additional markers, both closely linked and unlinked, were included in the cross to verify that the group of ascospores recovered was really derived from a single meiotic division.

This technique of recovering asci has only been used to a limited extent by the author. Only eight different strains were used in the six crosses (Table 2) and the biscuit strain was always used as the protoperithecial parent to avoid the web of aerial hyphae found in wild-type Neurospora. However, two other workers in this Department have used the method successfully with wild-type as the protoperithecial parent, in experiments concerning interference in a multiply-marked chromosome arm (Perkins, 1958) and in experiments with a translocation, where asci were classified with respect to their contents of ripe and aborted ascospores (Regnery, D. C., personal communication). On the other hand, Mrs B. Maling (personal communication) was unsuccessful in attempts to use the method in crosses of crisp $\mathrm{A} \times$ crisp a because no ascospores were shot from the perithecia.

The author wishes to thank Dr David D. Perkins for his valuable help during this study and in the preparation of the manuscript, and Mr George Pegelow for his technical assistance. This work was supported by a research grant (3840) from the National Science Foundation, U.S.A.

\section{REFERENCES}

Barratt, R. W., Newmeyer, D., Perkins, D. D. \& Garnjobst, L. (1954). Map construction in Neurospora crassa. Advanc. Genet. 6, 1.

Emerson, S. (1956). Notes on the identification of different causes of aberrant tetrad ratios in Saccharomyces. C.R. Lab. Carlsberg (Ser. physiol.), 26, 71.

Houlahan, M. B., Beadle, G. W. \& Calhoun, H. G. (1949). Linkage studies with biochemical mutants of Neurospora crassa. Genetics, 34, 493.

LINDEGREN, C. C. \& LINDEGREN, G. (1942). Locally specific patterns of chromatid and chromosome interference in Neurospora. Genetics, 27, 1.

Perkins, D. D. (1958). Crossing-over in linkage group I of Neurospora. Proc. 10th int. Congr. Genetics, 2, 214.

Shear, C. L. \& Dodge, B. O. (1927). Life histories and heterothallism of the red bread-mold fungi of the Monilia sitophila group. J. Agric. Res. 34, 1019.

Strickland, W. N., Perkins, D. D. \& Veatch, C. C. (1959). Linkage data for group V markers in Neurospora. Genetics, 44, 1120.

WestergaArd, M. \& Mitchell, H. K. (1947). Neurospora. V. A synthetic medium favouring sexual reproduction. Amer. J. Bot. 34, 573.

(Received 16 October 1959) 